\title{
Fulminant Brain Atrophy and Vasculitis on Vessel-Wall Imaging in Neuropsychiatric Lupus: Case Report and Literature Review
}

\author{
Mihaela Ionela SARBU1(D), Nicolae SARBU ${ }^{2,3}$ (D) \\ ${ }^{1}$ Saint Pierre University Hospital; Erasme Hospital, Rheumatology, Brussels, Belgium \\ ${ }^{2}$ Department of Neuroradiology, Erasme Hospital, Brussels, Belgium \\ ${ }^{3}$ Department of Neuroradiology, Faculty of Medicine, "Dunarea de Jos" University, Galati, Romania
}

\begin{abstract}
Brain atrophy, typically slowly progressive, is a hallmark of neuropsychiatric (NP) systemic lupus erythematosus (SLE). In this article, we report a case of a young female patient with previously diagnosed SLE, without known NPSLE, with abrupt neurological deterioration and rapidly progressive diffuse atrophy in a few months. A comprehensive diagnostic work-up and follow-up magnetic resonance imaging (MRI), including high-resolution advanced vessel-wall sequences, revealed underlying cerebral vasculitis. The novelty factors that the present report brings are the rapid progressive atrophy demonstrated on follow-up MRI in a patient with SLE, and the depiction of an underlying vasculitis on specific vessel-wall MRI techniques. We also reviewed the literature and discussed the main current applications of vessel-wall MRI sequences. The aim of the report is to recognize this dramatic form of presentation of NPSLE and the utility of the new MRI techniques for the diagnosis.

Keywords: Magnetic resonance imaging, neuropsychiatric systemic lupus erythematosus, vasculitis.
\end{abstract}

Brain atrophy is a hallmark of neuropsychiatric (NP) systemic lupus erythematosus (SLE), and probably the most important magnetic resonance imaging (MRI) biomarker for the diagnosis and prognosis of NPSLE. ${ }^{1,2}$ Atrophy in NPSLE is typically slowly progressive, over several years. Brain atrophy could be diffuse or could involve preferentially the fronto-temporal regions, with symmetrical involvement of gray and white matter. Underlying mechanisms of atrophy are not well understood, commonly incriminated factors being long-standing disease, activity and injury scores (Systemic Lupus Erythematosus Disease Activity Index, Systemic Lupus International Collaborating Clinics), complement deficiency, antiphospholipid (APL) antibodies, and long-term corticosteroid treatment. $^{2}$
In this article, we report the case of a young female patient previously diagnosed as SLE, who presented with important cognitive decline. A comprehensive diagnostic work-up and followup, including high-resolution specific vessel-wall imaging, established the diagnosis of cerebral vasculitis. MRI exams showed abrupt progression from normal brain to extremely severe atrophy in a few months. Rapid progressive cerebral atrophy was already recognized in NPSLE ${ }^{3}$ the novelty of this report being the underlying vasculitis demonstrated on vessel-wall MRI sequences as an underlying mechanism. Our purpose is to describe this form of onset of NPSLE and to make known the usefulness of the new MRI techniques in order to achieve the correct diagnosis.

Received: April 07, 2019 Accepted: July 16, 2019 Published online: January 08, 2020

Correspondence: Nicolae Sarbu, MD, PhD. Department of Neuroradiology, Erasme Hospital, 1070 Brussels, Belgium. Tel: 003225553111 e-mail: nicolae.sarbu@erasme.ulb.ac.be 


\section{CASE REPORT}

A 42-year-old female patient with SLE, previously well controlled, presented with extreme somnolence and cognitive decline. She was diagnosed with SLE seven years before, fulfilling the American College of Rheumatology (ACR) criteria. 4,5 General examination showed no abnormalities other than SLE-related, and neurological exams revealed no focal deficits. Subsequently, she was diagnosed with NPSLE, classified as cognitive disorder and acute confusional state accordingly to the ACR case definitions. ${ }^{6}$ The patient was receiving hydroxychloroquine and low-dose acetylsalicylic acid as chronic treatment. Laboratory investigations showed no abnormality in liver function or coagulation and the creatinine level was stable. The erythrocyte sedimentation rate was increased (63 mm/hour) as well as the C-reactive protein (34 mg/L $\mathrm{N}<10 \mathrm{mg} / \mathrm{L}$ ). Autoimmune panel showed positive antinuclear antibody, anti-double stranded deoxyribonucleic acid antibodies confirmed by Crithidia test, antiRo and anti-La antibodies and APL antibodies (lupus coagulant, immunoglobulin [Ig] M and IgG anticardiolipin). Complement fractions (C3, C4, $\mathrm{CH} 50)$ were low. Lumbar puncture showed leukocytosis with lymphocytosis (91\%), and elevated protein $(6 \mathrm{~g} / \mathrm{L})$ with predominantly IgG. Echocardiography, Holter electrocardiography, chest computed tomography and abdominal ultrasound were normal. Active systemic involvement was absent. Infection was ruled out, including comprehensive immunologic, serologic and cerebrospinal fluid tests for atypical microorganisms. A written informed consent was obtained from the patient.

Initial brain MRI, using a standard protocol, was normal (Figures 1a and 2a). Due to rapid deterioration of the neurological status, a followup MRI was performed three weeks later (Figures $1 \mathrm{~b}$ and $2 \mathrm{~b}$ ), including gadolinium administration and angiographic sequences. A mild periventricular nonspecific leukoencephalopathy and an incipient dilation of the supratentorial ventricles were observed. No abnormal contrastenhancement was present. MRI angiography and venography showed permeability of the vessels. The patient continued to deteriorate, in a span of several weeks, despite initiating high-dose intravenous methylprednisolone. The clinical suspicion of vasculitis was raised and a third MRI was performed, at 3.5 months from the onset, with a specific protocol including vessel-wall three-dimensional (3D)-high-resolution pre- and post-contrast black-blood sequences. This MRI showed severe progression of the leukoencephalopathy, and associated atrophy with marked dilation of the ventricles and the subarachnoid spaces (Figures 1c and 2c). High-resolution vessel-wall sequences showed multiple arterial segments with concentric contrast enhancement of the wall, of proximal and distal distribution (Figure 3), compatible with vasculitis. A treatment with high-dose intravenous cyclophosphamide was started. Clinical followup revealed a progressive improvement of the drowsiness with eventually stabilized neurological status, but the cognitive deficit was already extremely impaired. The fourth MRI exam, at seven months from the clinical onset, showed
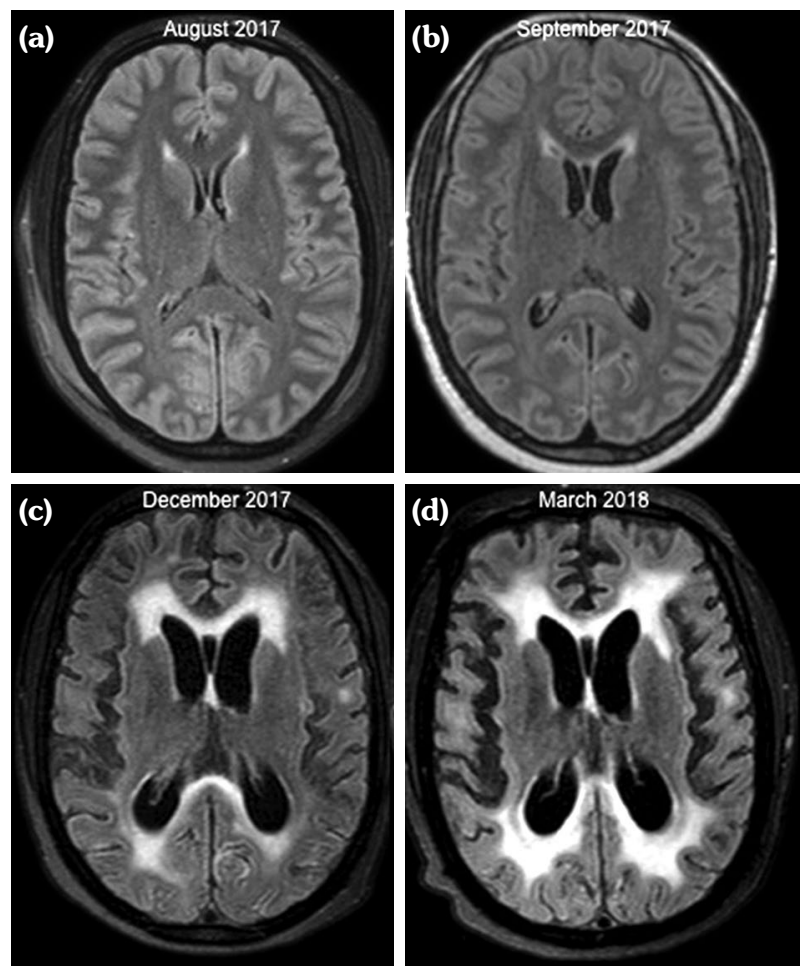

Figure 1. Progressive leukoencephalopathy and brain atrophy over a period of seven months. Initial fluidattenuated inversion recovery (FLAIR) sequence (a) shows no abnormalities. Follow-up FLAIR sequences (b-d) show development and progression of periventricular leukoencephalopathy with dilation of ventricular system and of cortical sulci. 

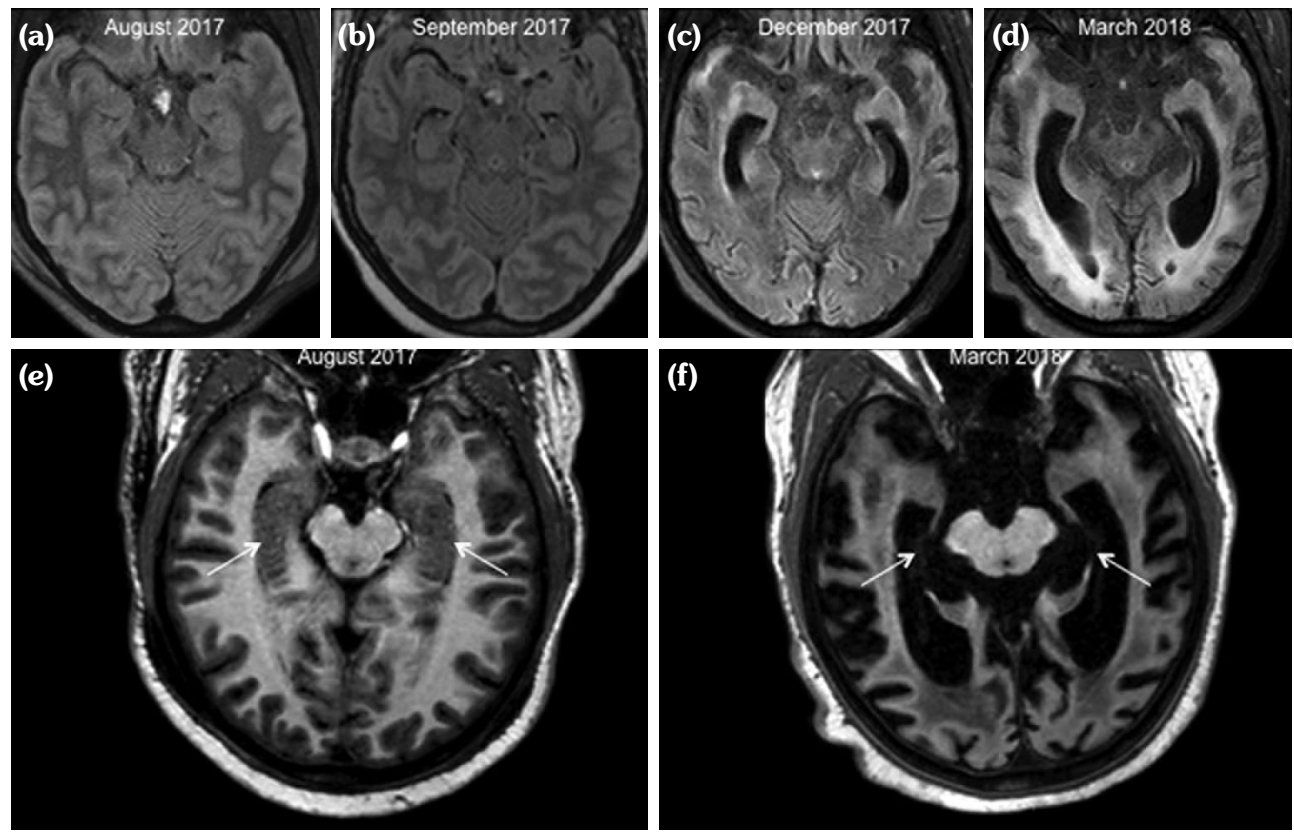

Figure 2. Severe atrophy of hippocampal formation and of temporal structures. Initial fluid-attenuated inversion recovery (FLAIR) image (a) shows normal volume and features of hippocampal and parahippocampal structures. Follow-up FLAIR sequence (b) shows incipient dilation of temporal horns of lateral ventricles, without other abnormalities. Next follow-up FLAIR sequences $(\mathbf{c}, \mathbf{d})$ show progressive important temporal leukoencephalopathy, severe dilation of lateral ventricles and severe volume loss of hippocampal and parahippocampal structures. T1-weighted sequences at clinical onset and seven months later better depict hippocampus, initially normal (arrows in e) and with severe "knife-blade" atrophy in last follow-up (arrows in $\mathbf{f}$ ).

even more severe atrophy (Figures $1 \mathrm{~d}$ and $2 \mathrm{~d}$ ). A comparative of the hippocampal formation, crucial for the memory circuit, showed normal features on the first MRI (Figure 2e) and marked "knife blade" atrophy on the last study (Figure 2f). Concomitant diseases, including cerebrovascular and cardiovascular events, were ruled out.

\section{DISCUSSION}

Neuropsychiatric SLE remains a major cause of morbidity in patients with SLE, despite all recent diagnostic and therapeutic advances. The most frequent MRI abnormalities in NPSLE are white matter lesions and brain atrophy, although the reported frequencies vary widely, probably reflecting the heterogeneity of the studied population. ${ }^{7,8}$ The presence of cortical atrophy was reported at a mean age of 42.5 years in NPSLE. ${ }^{7}$ Less frequently, NPSLE patients could present inflammatory lesions on MRI, which usually disappear after aggressive corticosteroid or immunosuppressive treatment. They were reported in $6.5 \%$ of patients, and were correlated with low complement levels, supporting an immune-mediated pathogenesis of NPSLE. ${ }^{9}$ This pattern has been related to primary cerebral vasculitis, which is however considered to be uncommon in SLE. $2,7,10$ Post-mortem histopathological studies reported cerebral vasculitis in less than $10 \%$ of NPSLE patients. ${ }^{9,11}$ Imaging findings of vasculitis in NPSLE were rarely described, when angiography exams revealed focal beadings and circumferential stenosis of arteries. ${ }^{12-14}$ On the other hand, a normal angiography cannot rule out vasculitis, and angiography exams are usually normal if small vessels are involved. ${ }^{11,14}$ Despite a low risk of complications, a brain biopsy is usually avoided, lacking of high sensitivity due to the sampling error with high false-negative 

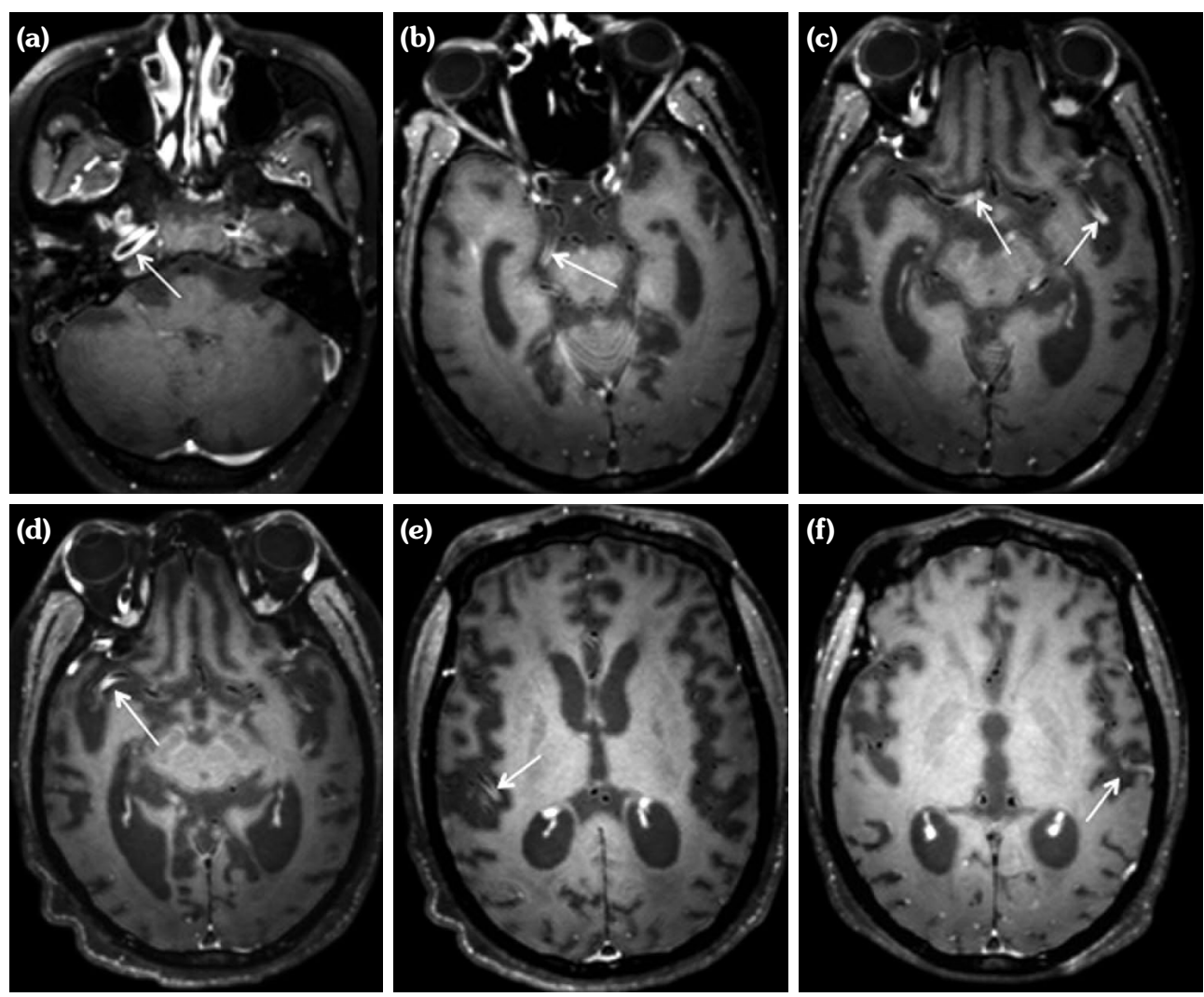

Figure 3. Cerebral vasculitis on high-resolution black-blood vessel-wall sequences. Circumferential vessel-wall strong contrast enhancement in multiple bilateral large proximal arteries (arrows in a-d) and small distal branches (arrows in e, f), demonstrating vasculitis.

rates. Therefore, no "gold standard" diagnostic test is accepted to date for the diagnosis of cerebral vasculitis. ${ }^{13,14}$ Recently, specific vessel-wall sequences emerged. This type of imaging requires a high-field MRI (3T), and the availability of high-resolution (around $0.5 \mathrm{~mm}$ isotropic) 3D-black-blood sequences, acquired pre- and post-gadolinium administration. ${ }^{14-17}$ Recent technological advances and guidelines were developed to optimize these sequences and to reduce the acquisition time on the one side ${ }^{15}$ and to provide expert consensus recommendations for their implementation in the current clinical practice on the other side. ${ }^{16,17}$ This vessel-wall imaging is increasingly used in other pathologies as aneurysms, dissection and strokes. Prospective comparative studies evaluating the feasibility of 3D-black-blood preand post-contrast sequences demonstrated that this technique accurately allows the diagnosis of vasculitis, and the intensity of contrast enhancement of the wall correlated with the mural inflammation. ${ }^{18}$ In the last year, several publications including these sequences reported vasculitis of different etiologies as varicellazoster, ${ }^{19}$ Sjögren syndrome, ${ }^{20}$ or primary vasculitis of the central nervous system. ${ }^{21} \mathrm{~A}$ recent study analyzed the vessel-wall imaging at 3 Tesla MRI in a cohort of SLE patients, and compared the data with a group of healthy controls. ${ }^{22}$ They demonstrated different types of vessel-wall focal lesions, more frequent in SLE group, particularly of the first segments of the middle cerebral artery. Moreover, vessel-wall lesions were associated with brain infarctions. Another recent report applied MRI angiography, vessel-wall imaging and transcranial Doppler and described multifocal stenosis of the intracranial arteries in two patients with SLE who presented non-aneurysmal subarachnoid hemorrhage. ${ }^{23}$ They concluded that the hemorrhage was due to vasculitis or to reversible cerebral vasoconstriction syndrome and that vessel-wall imaging is an important tool for the diagnosis. 
Previous case reports of cerebral vasculitis in SLE patients, documented on biopsy or angiography, described successful treatment with intravenous pulse cyclophosphamide in association or not with corticosteroids. ${ }^{11,13}$ Other immunosuppressive drugs as well as new treatments as biological therapies have also been used in SLE-related vasculitis, with variable results. ${ }^{14} \mathrm{~A}$ recent post-mortem brain study compared specimens from NPSLE patients with SLE patients and controls, and demonstrated that histopathological lesions in NPSLE represent a continuum, ranging from focal common vasculopathy to more specific diffuse vasculitis. ${ }^{9}$ They also showed that complement deposition was strongly associated with both NPSLE and SLE. Thus, they believe this deposition may be a link between autoantibodies and morphological lesions; therefore, complement inhibition may have novel therapeutic perspectives in NPSLE-related lesions as cerebral vasculitis.

To resume, the novelty factors that the present report brings are the rapid progressive atrophy demonstrated on MRI in a patient with NPSLE, and the depiction of underlying vasculitis on specific vessel-wall imaging. An accurate and timely diagnosis of cerebral vasculitis is fundamental for treatment initiation and outcome, giving the high morbidity and mortality of this condition. The physicians involved in the care of SLE patients should be aware of this type of presentation of NPSLE, and should understand the utility of the recent vessel-wall techniques. Vessel-wall MRI should probably be included in the work-up of SLE patients with unexplained neurological deficits or when a vasculitis could not be ruled out.

\section{Declaration of conflicting interests}

The authors declared no conflicts of interest with respect to the authorship and/or publication of this article.

\section{Funding}

The authors received no financial support for the research and/or authorship of this article.

\section{REFERENCES}

1. Postal M, Lapa AT, Reis F, Rittner L, Appenzeller $\mathrm{S}$. Magnetic resonance imaging in neuropsychiatric systemic lupus erythematosus: current state of the art and novel approaches. Lupus 2017;26:517-21.

2. Sarbu N, Sarbu MI, Bargallo N, Cervera R. Future perspectives in the diagnosis of neuropsychiatric lupus by advanced magnetic resonance imaging techniques. Curr Rheumatol Rev 2018;14:213-8.

3. Katsumata Y, Kawaguchi Y, Yamanaka H. Rapid progressive cerebral atrophy in systemic lupus erythematosus. J Rheumatol 2011;38:2689.

4. Tan EM, Cohen AS, Fries JF, Masi AT, McShane DJ, Rothfield NF, et al. The 1982 revised criteria for the classification of systemic lupus erythematosus. Arthritis Rheum 1982;25:1271-7.

5. Hochberg MC. Updating the American College of Rheumatology revised criteria for the classification of systemic lupus erythematosus. Arthritis Rheum 1997;40:1725.

6. American College of Rheumatology (ACR) ad hoc committee on neuropsychiatric lupus: The American College of Rheumatology nomenclature and case definitions for neuropsychiatric lupus syndrome. Arthritis Rheum 1999;42:599-608.

7. Sarbu N, Alobeidi F, Toledano P, Espinosa G, Giles I, Rahman A, et al. Brain abnormalities in newly diagnosed neuropsychiatric lupus: systematic MRI approach and correlation with clinical and laboratory data in a large multicenter cohort. Autoimmun Rev 2015;14:153-9.

8. Roura E, Sarbu N, Oliver A, Valverde S, González-Villà $\mathrm{S}$, Cervera R, et al. Automated Detection of Lupus White Matter Lesions in MRI. Front Neuroinform 2016;10:33.

9. Cohen D, Rijnink EC, Nabuurs RJ, Steup-Beekman GM, Versluis MJ, Emmer BJ, et al. Brain histopathology in patients with systemic lupus erythematosus: identification of lesions associated with clinical neuropsychiatric lupus syndromes and the role of complement. Rheumatology (Oxford) 2017;56:77-86.

10. Sarbu N, Toledano P, Calvo A, Roura E, Sarbu MI, Espinosa G, et al. Advanced MRI techniques: biomarkers in neuropsychiatric lupus. Lupus 2017;26:510-6.

11. Rowshani AT, Remans P, Rozemuller A, Tak PP. Cerebral vasculitis as a primary manifestation of systemic lupus erythematosus. Ann Rheum Dis 2005;64:784-6.

12. Meroni PL, Raschi E, Camera M, Testoni C, Nicoletti $\mathrm{F}$, Tincani $\mathrm{A}$, et al. Endothelial activation by aPL: a potential pathogenetic mechanism for the clinical manifestations of the syndrome. $\mathrm{J}$ Autoimmun 2000;15:237-40.

13. Rizos T, Siegelin M, Hähnel S, Storch-Hagenlocher B, Hug A. Fulminant onset of cerebral immunocomplex vasculitis as first manifestation of neuropsychiatric systemic lupus erythematosus (NPSLE). Lupus 2009;18:361-3.

14. Barile-Fabris L, Hernández-Cabrera MF, BarraganGarfias JA. Vasculitis in systemic lupus erythematosus. Curr Rheumatol Rep 2014;16:440. 
15. Zhu C, Tian B, Chen L, Eisenmenger L, Raithel E, Forman $\mathrm{C}$, et al. Accelerated whole brain intracranial vessel wall imaging using black blood fast spin echo with compressed sensing (CS-SPACE). MAGMA 2018;31:457-67.

16. Mandell DM, Mossa-Basha M, Qiao Y, Hess CP, Hui F, Matouk C, et al. Intracranial Vessel Wall MRI: Principles and Expert Consensus Recommendations of the American Society of Neuroradiology. AJNR Am J Neuroradiol 2017;38:218-29.

17. Tan HW, Chen X, Maingard J, Barras CD, Logan C, Thijs $\mathrm{V}$, et al. Intracranial Vessel Wall Imaging with Magnetic Resonance Imaging: Current Techniques and Applications. World Neurosurg 2018;112:186-98.

18. Treitl KM, Maurus S, Sommer NN, KooijmanKurfuerst H, Coppenrath E, Treitl M, et al. 3D-blackblood 3T-MRI for the diagnosis of thoracic large vessel vasculitis: A feasibility study. Eur Radiol 2017;27:2119-128.

19. Waters MJ, Kleinig T. Black-blood magnetic resonance imaging demonstrates varicella zoster vasculitis. Intern Med J 2018;48:1408-10

20. Unnikrishnan G, Hiremath N, Chandrasekharan K, Sreedharan SE, Sylaja PN. Cerebral large-vessel vasculitis in Sjogren's syndrome: Utility of highresolution magnetic resonance vessel wall imaging. $\mathrm{J}$ Clin Neurol 2018;14:588-90.

21. Tsivgoulis G, Papadimitropoulos GN, Lachanis S, Palaiodimou L, Zompola C, Antonellou R, et al. High-resolution intracranial vessel wall imaging in monitoring treatment response in primary CNS angiitis. Neurologist 2018;23:188-90.

22. Ide S, Kakeda S, Miyata M, Iwata S, Ohkubo N, Nakayamada $S$, et al. Intracranial vessel wall lesions in patients with systematic lupus erythematosus. J Magn Reson Imaging 2018;48:1237-46.

23. Lee JY, Kang S, Park JS, Jo SJ. Prevalence of psoriasis in Korea: A population-based epidemiological study using the korean national health insurance database. Ann Dermatol 2017;29:761-7. 